in the way depicted or whilst the injected fluid is retained in the ordinary way. The clamp is then pinned to the inside of the inside shirt and the patient rests or goes about with that quantity of fluid, and of proper strength which he finds not uncomfortable, distending his anterior urethra. I find that even this part of the urethra is capable of definite absorptive power, in the absence of any fluid passing the urethral sphincter. I am indebted to $\mathrm{Mr}$. Lewis of Messrs. Allen and Hanburys for carrying out my ideas.

Finsbury-pavement, E.C. JaMES MaCMUnN.

\section{A TONGUE DEPRESSOR FOR EXPOSING THE TONSIL.}

THIS instrument is a modification of Jaenicke's tongue depressor. As shown in the illustration, it consists of an angular tongue depressor having on one side a small double blunt hook fixed at right angles to the blade. The instrument is reversible and by having a hook attached to either blade, as shown in the accompanying figure, it can be used for both tonsils. By engaging the hook in the anterior fancial pillar or in the plica triangularis and drawing the same gently forwards and outwards the surface of the tonsil and the opening of the supra-tonsillar fossa can be fully

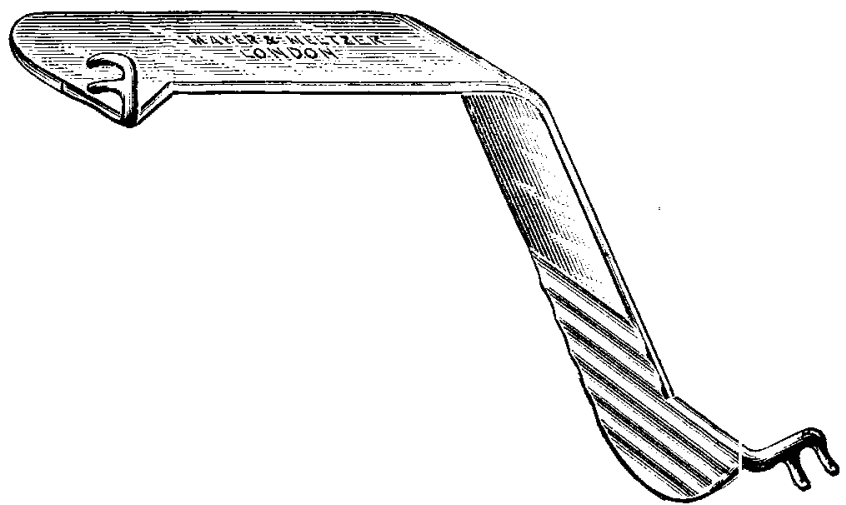

exposed. As this tongue depressor requires only one hand for its use it leaves the other free for the application of a probe or any other instrument to the tonsil. It may be used with or without cocaine. I have found this instrument of especial use in cases of disease in non-projecting tonsils in adults. It has been made for me by Messrs. Mayer and Meltzer, 71, Great Portland-street, London, W.

Brighton.

E. Cresswell Baber.

\section{Looking JBack.}

FROM

THE LANCET, SATURDAY, APRIL 16, 1825.

\author{
LECTURES \\ ON THE \\ PRINCIPLES AND PRACTICE OF PHYSIC, \\ BY DR. ARMSTRONG.
}

Theatre of Anatomy, Webb Street.

\section{LECTURE $21{ }^{1}$}

Look at nature, and you will find that gout is no more peculiar than inflammation at the end of the nose is peculiar, for that, like the inflammation about the great toe, has generally one common origin-some disturbance in the stomach or bowels; but because some writers, in the dark ages, chose to say it arose from some peculiar and unknown cause, it has been still so considered, even from the time of GaLEN, who avowed that gout

1 Only a portion of the lecture has been transcribed. depended on some peculiar condition of the fluids:nay, I know of no subject on which common sense, and the common principles of pathology, have been so abandoned as on this, even in some huge modern volumes published on the disorder. If I were to speak from my own observation, then, I would say, that gout is nothing more than an inflammatory affection which is seated in the structures adjacent to joints, and which is always preceded, or attended, by some disorder of the stomach, liver, or boncls. You have a right to ask, what is that disorder? and, as I am bound to answer the question, I say, that the disorder is various; sometimes it is merely a slight degree of irritation on the mucous surface of the stomach, what I before explained under the term local simple excitement ; but in some cases the irritation is really a low degree of infammation in that membrane, or a similar condition exists, separately or combinedly, on the same continuous structure of the small intestines. The affection of the liver is, perhaps, mostly congestive, but occasionally attended by an obscure indication of inflammation, as pressure reveals, the bile in both cases being defective or depraved. Generally too there is torpor of the colon, but now and then even irritation on its mucous lining; and the skin is commonly out of order. These affections are sometimes separate, at other times co-existent, as I have before stated; but they invariably precede or attend the external inflammation called gout, which may be considered an effect of the internal affection, and which may be acute, subacute, or chronic. Where it assumes an acute or subacute character, it is sufficient to disturb the whole nervous system, and that disturbance again affects the vascular system, so that the animal heat on the surface and the heart's action become increased, or that state usually termed fever is established; but when the inflammation is chronic it seldom excites fever. This affection arises then from what we call sympathy, but we do not know in what mode precisely, and therefore I merely use the word sympathy to express the fact of the connexion which exists between one part of the body and another, and the knowledge of the fact is of great pathological importance.

\section{FOREIGN DEPARTMENT.}

ANALYSIS OF FOREIGN MEDICAL JOURNALS.

FROM MIAGENDIE'S JOURNAL DE PHYSIOLOGIE.

Observations on Diabetes Mcllitus, by MM. TAUQUELIN and De SEgalas D'Ecthepare. ${ }^{2}$

A WOMAN, in her fiftieth year, came to request the advice of Dr. Asselin on this complaint, who thought it a fit opportunity to ascertain if sugar exists in the blood of persons affected with this disease. In consequence of the inflammatory symptoms having required two large bleedings, the blood drawn was analysed with the most scrupulous attention. It was impossible to discover in it one atom of sugar, notwithstanding the urine voided, amounting to nine or ten pints daily, contained a seventh of this substance. The saliva also, examined at two different times, left not the slightest trace of it. Urea was proposed as a remedy, and the patient took it for several days; when the urine was again analysed, the quantity of sugar was rather increased.

2 The details of the different analyses have not been transcribed.

Some Novel Patents.- An American inventor has patented what he calls a "bath and fever machine." It consists of a closed box or chamber supported on feet and provided with steam and hot-air pipes leading from an external heating apparatus, "the discharge ends of the pipes being closely disposed to the foot portion of the slatted top and gradually spaced away therefrom towards its top, thereby to cause an increase in the heat from the head of the patient to his feet." A patent has also been obtained for a hog-nose cutter which is described as "an implement for removing the rooting portions of hogs' noses, comprising a pair of handles pivoted together" ; further constructional details are then given. Specifications and drawings of both these contrivances will be found in the Official Gazette of the United States Patent Office issued on March 17th. 


\section{THE LANCE'.}

gency of opinion exists as to what action should be taken in such circumstances. Some pharmacists consider that the carbonate of bismuth should be substituted, whilst others are of opinion that the mixture should be so treated that all, or most of, the chemical action which ensues is over before the resulting compound is sent out. We think that both these courses are wrong, agreeing in the matter with Mr MoEwaN. Although the two salts of bismuth are generally considered to be practically identical in their medicinal properties, yet some practitioners believe that the carbonate is the blander and the less astringent of the two. Further, if heat is employed to hasten the action referred to the patient would receive a larger dose of sodium nitrate. It is preferable that the prescription should be dispensed as written or referred to the writer. We may add that it is not a common event for an explosion to occur as the result of the reaction between the bismuth and sodium salts, and besides being rare the explosion, as a rule, only amounts to the blowing out of the cork from the bottle. The question of dosage, again, frequently taxes the dispenser's powers of discretion. Mr. MCEWAN instances the following: "Sodii bromid., 10 grs.; ammon. bromid., 10 grs. ; tinct. card. co., $20 \mathrm{~min}$. ; syr. aurant., $1 \mathrm{dr}$.; aq. ad $8 \mathrm{oz}$. $1 \mathrm{oz}$. ter die post cib." The difficulty in which the dispenser would be placed in making up this prescription can be easily appreciated. We should feel disposed to think that the practitioner had intended to write directions for one dose only and had then inadvertently made up the mixture to 8 ounces instead of to 1 ounce. Nevertheless, as it is possible that only minute doses of the bromides were designed perhaps Mr. McEWAN is right in his opinion that there was no justification for interference. It is, however, one of those cases in which the prescriber might well have been communicated with. In consequence of the want of practical dispensary experience on the part of medical students when they pass into actual practice those who wish to prescribe a pill leave the choice of a suitable excipient very often to the discretion of the dispenser. $\mathrm{Mr}$. MoEWAN's remarks on this subject are instructive. He says that, according to his experience, the course which we have just mentioned is adopted in about 60 per cent. of the prescriptions met with; in about 35 per cent. of the cases a suitable excipient is ordered, but it may be either in excess or slightly deficient; and in 5 per cent. of instances the excipient which is ordered is unsuitable. It must be remembered, however, that in a large number of cases the excipient possesses no therapeutical properties and the choice may, therefore, as a rule, be safely left to the discretion of the dispenser. Mr. McEWAN says little about poisonous doses being ordered by mistake. It was scarcely necessary to do so, as the course to be followed in such an event is clear-namely, to draw the attention of the prescriber to the amourt ordered.

It is obvious that occasionally alterations in prescriptions are absolutely necessary; this being so, if in the exercise of his discretion a dispenser finds it expedient to modify a prescription we deem it highly advisable that the writer of it should be informed of the fact. Serious mistakes in prescriptions are very rare, and the practitioner, far from resenting the interference, is always grateful to the dispenser for any error that is pointed out. The medical man nate. Apparently amongst dispensers considerable diver- 